NIKHEF-H/94-02

February 2, 1994

\title{
A new perspective on electroweak strings
}

\author{
F. R. Klinkhamer \\ CHEAF/NIKHEF-H \\ Postbus 41882 \\ NL-1009 DB Amsterdam \\ The Netherlands \\ and \\ P. Olesen \\ The Niels Bohr Institute \\ University of Copenhagen \\ Blegdamsvej 17 \\ DK-2100 Copenhagen \\ Denmark
}

\begin{abstract}
The vortex solution ( $Z$-string) of the electroweak interactions can be interpreted as the 2-dimensional sphaleron at the top of a non-contractible sphere. The same holds for another type of solution, the $W$-string.
\end{abstract}




\section{Introduction}

It has been known for a long time that the vortex solution [1] of the Abelian Higgs model may be embedded [2] in the electroweak standard model, where it excites the $Z^{0}$ and Higgs fields. Recently, the stability properties of this electroweak vortex solution ( $Z$-string) have been investigated numerically [3]. The $Z$-string solution turns out to be metastable for values of the weak mixing angle $\theta_{w}$ very close to $\pi / 2$ and small enough Higgs mass. For the physical mixing angle $\theta_{w} \sim \pi / 6$, however, the solution is unstable, most likely for all values of the Higgs mass. The existence and potential stabilty of the $Z$-string for $\theta_{w} \rightarrow \pi / 2$ are well understood [ [ 6 , 5]. In this article we start from $\theta_{w}=0$ and "explain" the existence and instability of the solution, by showing analytically that, for $\theta_{w} \leq \pi / 4$ at least, the $Z$-string is the 2-dimensional sphaleron at the top of a non-contractible sphere in configuration space. Recall that, generally speaking, a sphaleron denotes a static, but unstable, finite energy solution of the classical field equations, where the theory may be defined in any number of spatial dimensions.

The outline of this article is as follows. In Sect. 2 we establish our notation. In

Sect. 3 we construct a non-contractible sphere of 2-dimensional configurations of the fields and in Sect. 4 we show that, for small enough values of $\theta_{w}$, its maximum corresponds to the $Z$-string solution. We also construct in Sects. 3 and 4 another, simpler non-contractible sphere, which is related to a different solution, the $W$-string [6], with larger energy per unit length than the $Z$-string. The main thrust of this paper is analytical, but, for completeness, we present in Sect. 4 some numerical results for both types of solutions and their corresponding non-contractible spheres. In Sect. 5, finally, we summarize our main observations and present a simple picture of configuration space.

\section{Notation}

We consider static classical fields in the bosonic sector of the electroweak standard model, namely the $S U(2)$ gauge field $W_{m} \equiv W_{m}^{a} \tau^{a}$, the $U(1)$ hypercharge gauge field $B_{m}$ and the complex Higgs doublet field $\Phi$. The energy functional of these fields is given by

$$
E=\int_{\mathrm{R}^{3}} d^{3} x\left[\frac{1}{4 g^{2}}\left(W_{m n}^{a}\right)^{2}+\frac{1}{4 g^{\prime 2}}\left(B_{m n}\right)^{2}+\left|D_{m} \Phi\right|^{2}+\lambda\left(|\Phi|^{2}-v^{2} / 2\right)^{2}\right]
$$


with the following definitions for the field strengths and covariant derivatives

$$
\begin{aligned}
W_{m n} & \equiv W_{m n}^{a} \tau^{a} \equiv \partial_{m} W_{n}-\partial_{n} W_{m}+\left[W_{m}, W_{n}\right] \\
B_{m n} & \equiv \partial_{m} B_{n}-\partial_{n} B_{m} \\
D_{m} \Phi & \equiv\left(\partial_{m}+W_{m}^{a} \frac{\sigma^{a}}{2 i}+B_{m} \frac{1}{2 i}\right) \Phi
\end{aligned}
$$

where the indices run over the values $1,2,3$, and $\tau^{a} \equiv \sigma^{a} /(2 i)$ are the $S U(2)$ generators, with $\sigma^{a}$ the standard Pauli matrices. The semiclassical masses of the $W^{ \pm}$and $Z^{0}$ vector bosons are $M_{W}=\frac{1}{2} g v$ and $M_{Z}=M_{W} / \cos \theta_{w}$, with the weak mixing angle $\theta_{w}$ defined as $\tan \theta_{w} \equiv g^{\prime} / g$. The mass of the physical Higgs scalar is $M_{H}=\sqrt{8 \lambda / g^{2}} M_{W}$.

In this article we are interested in axisymmetric field configurations which are constant along the symmetry axis. We use the standard cylindrical coordinates $\rho$, $\phi$ and $z$, defined in terms of the cartesian coordinates by $\left(x_{1}, x_{2}, x_{3}\right) \equiv(\rho \cos \phi$, $\rho \sin \phi, z)$. Making all distances dimensionless $\left(\tilde{\rho} \equiv \rho M_{Z}\right.$ etc. , and dropping the tildes afterwards) we then write for the total energy of these fields

$$
E \equiv \frac{2 \pi v \cos \theta_{w}}{g} \int_{-\infty}^{\infty} d z \epsilon=\frac{2 \pi v \cos \theta_{w}}{g} \int_{-\infty}^{\infty} d z \int_{0}^{\infty} d \rho \rho e(\rho),
$$

with $e$ the dimensionless energy density and $\epsilon$ the dimensionless energy per unit length (string tension). The total energy $E$ is infinite for the configurations we consider and, instead, we focus on the 2-dimensional energy $\epsilon$, which should be finite. Specifically, we investigate the topology of the space of finite $\epsilon$ configurations and in the next section we will take two slices through this infinitely dimensional configuration space. []

\section{Non-contractible spheres}

In this section we present two non-contractible spheres of 2-dimensional axisymmetric field configurations, i. e. configurations that depend on $\rho$ and that have vanishing $z$-components of the gauge fields. They differ in that the first non-contractible sphere (NCS) has $S U(2)$ gauge fields only, whereas the second NCS excites both the $S U(2)$ and $U(1)$ gauge fields. We will call the first non-contractible sphere the $W$-NCS and the second the $Z$-NCS, because the first has at the top $W^{ \pm}$gauge fields whereas the second has a $Z^{0}$ gauge field instead.

\footnotetext{
${ }^{1}$ We consider in this article the minimal standard model, but we expect our topological considerations to have more general validity.
} 


\section{1 $W$-NCS}

The NCS is parametrized by the square $\mu, \nu \in[-\pi,+\pi]$, with the boundary $|\mu|=\pi$ or $|\nu|=\pi$ corresponding to the classical vacuum. Writing $[\mu \nu] \equiv \max (|\mu|,|\nu|)$ and using differential forms, the field configurations of the NCS are

$$
\begin{aligned}
\pi / 2 \leq[\mu \nu] \leq \pi \quad: \quad & W=0 \\
B & =0 \\
\Phi & =(1-(1-\tilde{h}) \sin [\mu \nu]) \frac{v}{\sqrt{2}}\left(\begin{array}{l}
0 \\
1
\end{array}\right) \\
0 \leq[\mu \nu]<\pi / 2 \quad: \quad W & =-\tilde{f} \tilde{\Omega} \mathrm{d} \tilde{U} \tilde{U}^{-1} \tilde{\Omega}^{-1} \\
B & =0 \\
\Phi & =\tilde{h} \frac{v}{\sqrt{2}} \tilde{\Omega} \tilde{U}\left(\begin{array}{l}
0 \\
1
\end{array}\right),
\end{aligned}
$$

with the $S U(2)$ matrices

$$
\tilde{U}(\mu, \nu, \phi)=\left(\begin{array}{l}
\sin \mu \\
\cos \mu \sin \nu \\
\cos \mu \cos \nu \sin \phi \\
\cos \mu \cos \nu \cos \phi
\end{array}\right) \cdot\left(\begin{array}{c}
-i \sigma_{2} \\
-i \sigma_{3} \\
1 \\
-i \sigma_{1}
\end{array}\right)
$$

and

$$
\tilde{\Omega}=\tilde{U}(\mu, \nu, 0)^{-1}=\sin \mu i \sigma_{2}+\cos \mu \sin \nu i \sigma_{3}+\cos \mu \cos \nu i \sigma_{1} .
$$

The matrix $\tilde{U}$ gives a mapping of the 3 -sphere into $S U(2)$ with unit winding number and $\tilde{\Omega}$ implements a global gauge transformation of the fields. The axial functions $\tilde{f}(\rho)$ and $\tilde{h}(\rho)$ in (3) have the following boundary conditions

$$
\begin{array}{r}
\lim _{\rho \rightarrow \infty} \tilde{f}, \tilde{h}=1 \\
\tilde{f}(0)=\tilde{h}(0)=0,
\end{array}
$$

which guarantee, respectively, vanishing energy density at infinity and regularity of the fields at the core. Note that the 2-dimensional fields of the NCS (3) are in the radial gauge $\left(W_{\rho}=B_{\rho}=0\right)$ and that, in general, the remaining global gauge freedom is fixed by the value of the Higgs field $\Phi$ at the point $\phi=0, \rho \rightarrow \infty$.

The dimensionless energy density (21) becomes for $0 \leq[\mu \nu]<\pi / 2$

$$
\begin{aligned}
e= & \cos ^{2} \mu \cos ^{2} \nu\left[\cos ^{-2} \theta_{w} \rho^{-2}\left(\partial_{\rho} \tilde{f}\right)^{2}+\rho^{-2} \tilde{h}^{2}(1-\tilde{f})^{2}\right]+ \\
& \left(\partial_{\rho} \tilde{h}\right)^{2}+\frac{1}{4}\left(\frac{M_{H}}{M_{Z}}\right)^{2}\left(\tilde{h}^{2}-1\right)^{2}
\end{aligned}
$$


and for $\pi / 2 \leq[\mu \nu] \leq \pi$

$$
e=\left(\partial_{\rho} \tilde{k}\right)^{2}+\frac{1}{4}\left(\frac{M_{H}}{M_{Z}}\right)^{2}\left(\tilde{k}^{2}-1\right)^{2}
$$

with $\tilde{k} \equiv 1-(1-\tilde{h}) \sin [\mu \nu]$. Manifestly, the maximum of the 2-dimensional energy $\epsilon(\mu, \nu)$ occurs at $\mu=\nu=0$. I The maximum energy $\epsilon(0,0)$ can be minimized by solving the variational equations from (2, 7), and the resulting functions are denoted by $\overline{\tilde{f}}$ and $\overline{\tilde{h}}$. This procedure is called a minimax procedure.

\section{$3.2 \quad Z$-NCS}

The construction of the second NCS starts from a "rotated" version of the $S U(2)$ matrix (4), which, at $\mu=\nu=0$ in particular, allows for the excitation of a $U(1)$ gauge field. Using the same notation as before $\left(\tau^{a} \equiv \sigma^{a} /(2 i)\right)$, the field configurations of this NCS are

$$
\begin{aligned}
\pi / 2 \leq[\mu \nu] \leq \pi \quad: \quad & W=0 \\
B & =0 \\
\Phi & =(1-(1-h) \sin [\mu \nu]) \frac{v}{\sqrt{2}}\left(\begin{array}{l}
0 \\
1
\end{array}\right) \\
0 \leq[\mu \nu]<\pi / 2 \quad: \quad W & =-f G^{a} \tau^{a} \\
B & =f \sin ^{2} \theta_{w} F^{3} \\
\Phi & =h \frac{v}{\sqrt{2}} \Omega U\left(\begin{array}{l}
0 \\
1
\end{array}\right),
\end{aligned}
$$

with the following Lie algebra valued 1-forms

$$
\begin{aligned}
F^{a} \tau^{a} & \equiv U^{-1} \mathrm{~d} U \\
G^{a} \tau^{a} & \equiv \Omega U\left[F^{1} \tau^{1}+F^{2} \tau^{2}+\cos ^{2} \theta_{w} F^{3} \tau^{3}\right] U^{-1} \Omega^{-1}
\end{aligned}
$$

and $S U(2)$ matrices

$$
\begin{aligned}
U(\mu, \nu, \phi) & =\left(\begin{array}{l}
\sin \mu \\
\cos \mu \sin \nu \\
\cos \mu \cos \nu \sin \phi \\
\cos \mu \cos \nu \cos \phi
\end{array}\right) \cdot\left(\begin{array}{c}
-i \sigma_{1} \\
-i \sigma_{2} \\
-i \sigma_{3} \\
1
\end{array}\right) \\
\Omega & =U(\mu, \nu, 0)^{-1} .
\end{aligned}
$$

\footnotetext{
${ }^{2}$ In order to have a manifest maximum the ansatz (3) is split into a part that "rotates" the gauge fields (taking the Higgs along for the ride) and a part that ties the Higgs field to its vacuum value, rather than performing the two operations together.
} 
The axial functions $f(\rho)$ and $h(\rho)$ in (9) have the usual boundary conditions

$$
\begin{aligned}
\lim _{\rho \rightarrow \infty} f, h & =1 \\
f(0)=h(0) & =0,
\end{aligned}
$$

in order to have smooth fields with finite 2-dimensional energy $\epsilon$.

The dimensionless energy density (21) becomes for $0 \leq[\mu \nu]<\pi / 2$

$$
\begin{aligned}
e= & \cos ^{2} \mu \cos ^{2} \nu\left(1-\cos ^{2} \mu \cos ^{2} \nu \sin ^{2} \theta_{w}\right) \cos ^{-2} \theta_{w}\left[\rho^{-2}\left(\partial_{\rho} f\right)^{2}\right]+ \\
& \cos ^{2} \mu \cos ^{2} \nu\left[\rho^{-2} h^{2}(1-f)^{2}\right]+\left(\partial_{\rho} h\right)^{2}+\frac{1}{4}\left(\frac{M_{H}}{M_{Z}}\right)^{2}\left(h^{2}-1\right)^{2}
\end{aligned}
$$

and for $\pi / 2 \leq[\mu \nu] \leq \pi$

$$
e=\left(\partial_{\rho} k\right)^{2}+\frac{1}{4}\left(\frac{M_{H}}{M_{Z}}\right)^{2}\left(k^{2}-1\right)^{2},
$$

with $k \equiv 1-(1-h) \sin [\mu \nu]$. For small enough values of $\theta_{w}$, the maximum of the 2-dimensional energy $\epsilon(\mu, \nu)$ occurs at $\mu=\nu=0$. Again, the minimax procedure is to solve the variational equations from (2, 14) at $\mu=\nu=0$, and the resulting functions are denoted by $\bar{f}$ and $\bar{h}$. In the next section we will investigate the optimal maximum configuration of this NCS further.

\section{Sphalerons}

By now it is well known that the topology of the configuration space of the electroweak standard model gives rise to non-trivial classical solutions. Specifically, the existence of non-contractible loops and non-contractible spheres of 3-dimensional configurations of the bosonic fields leads to, respectively, the sphaleron S [7] and the sphaleron $S^{\star}[8]$. In the same way we may expect the non-contractible spheres of 2-dimensional configurations, as constructed in the previous section, to be related to non-trivial string-like classical solutions. In this Section we will show that these solutions correspond to the known $W$ - and $Z$-string solutions. Furthermore, we will demonstrate that these solutions are at the top of their respective non-contractible spheres, so that they are really sphalerons (for the $Z$-string this holds provided $\theta_{w}$ is small enough).

\section{1 $W$-string}

The maximum configuration of the $W$-NCS (3) reproduces, up to a global gauge transformation, to the so-called $W$-string solution [6], which excites the $W^{ \pm} \equiv$ 
$\left(W^{1} \mp i W^{2}\right) / \sqrt{2}$ gauge fields. More precisely, the minimax procedure over the $W$ NCS gives the $W$-string solution with axial functions $f_{W}=\overline{\tilde{f}}$ and $h_{W}=\overline{\tilde{h}}$. From the energy density (7) we then find the energy profile over the $W$-NCS for $0 \leq[\mu \nu]<\pi / 2$

$$
\epsilon(\mu, \nu)=\epsilon_{W}\left[\cos ^{2} \mu \cos ^{2} \nu(\tilde{a}+\tilde{b})+\tilde{c}+\tilde{d}\right],
$$

in terms of the following integrals

$$
\begin{aligned}
& \tilde{a}+\tilde{b} \equiv \epsilon_{W}^{-1} \int_{0}^{\infty} d \rho \rho\left[\cos ^{-2} \theta_{w} \rho^{-2}\left(\partial_{\rho} f_{W}\right)^{2}+\rho^{-2} h_{W}^{2}\left(1-f_{W}\right)^{2}\right] \\
& \tilde{c}+\tilde{d} \equiv \epsilon_{W}^{-1} \int_{0}^{\infty} d \rho \rho\left[\left(\partial_{\rho} h_{W}\right)^{2}+1 / 4\left(M_{H} / M_{Z}\right)^{2}\left(h_{W}^{2}-1\right)^{2}\right],
\end{aligned}
$$

which add up to 1 . The energy $\epsilon(\mu, \nu)$ for $\pi / 2 \leq[\mu \nu] \leq \pi$ decreases monotonically with $[\mu \nu]$, as follows by inspection of (8). In table 1 we give some numerical results for the energy $\epsilon_{W}$ and the integrals (17). The relation $\epsilon_{W}\left(\sin ^{2} \theta_{w}=3 / 4 ; M_{H} / M_{Z}\right)=$ $\epsilon_{W}\left(\sin ^{2} \theta_{w}=0 ; 2 M_{H} / M_{Z}\right)$ displayed in this table follows directly from the scaling properties of $\epsilon_{W}$ as given by (17). The dimensionless energy $\epsilon_{W}$ is, in fact, a function of $\cos ^{-1} \theta_{w} M_{H} / M_{Z}=M_{H} / M_{W}$ only. In short, we have $\epsilon_{W}=\epsilon_{W}\left(M_{H} / M_{W}\right)$.

From the energy profile (16) we conclude immediately that the $W$-string is the 2-dimensional sphaleron of a non-contractible sphere, with at least two negative modes. This holds for arbitrary values of $\theta_{w}$ and $M_{H} / M_{Z}$. We turn now to the other type of string solution, where the situation is more complicated.

\section{$4.2 \quad Z$-string}

First, recall that the fields of the electroweak vortex solution ( $Z$-string) «1, 2] can be written in the following form

$$
\begin{aligned}
W & =-\cos ^{2} \theta_{w} f_{Z} \mathrm{~d} V V^{-1} \\
B & =-\tan ^{2} \theta_{w} W^{3} \\
\Phi & =h_{Z} \frac{v}{\sqrt{2}} V\left(\begin{array}{l}
0 \\
1
\end{array}\right)
\end{aligned}
$$

where the $S U(2)$ matrix $V$ is defined in terms of the matrix $U(\mu, \nu, \phi)$ of eq. (12) by

$$
V \equiv U(0,0, \phi)
$$

and the functions $f_{Z}$ and $h_{Z}$ are the solutions of the field equations with boundary conditions (13). The corresponding energy density

$$
e_{Z}=\rho^{-2}\left(\partial_{\rho} f_{Z}\right)^{2}+\rho^{-2} h_{Z}^{2}\left(1-f_{Z}\right)^{2}+\left(\partial_{\rho} h_{Z}\right)^{2}+\frac{1}{4}\left(\frac{M_{H}}{M_{Z}}\right)^{2}\left(h_{Z}^{2}-1\right)^{2}
$$


is independent of the value of the weak mixing angle $\theta_{w}$. This energy density gives the dimensionless $Z$-string energy $\epsilon_{Z}=\epsilon_{Z}\left(M_{H} / M_{Z}\right)$, which is related to the $W$ string energy in the following simple way

$$
\epsilon_{Z}\left(M_{H} / M_{Z}\right)=\epsilon_{W}\left(M_{H} / M_{W}\right) .
$$

The relation (21) is not entirely unexpected, considering the fields involved in both types of string solutions. Their stability properties, however, may be different, depending on the value of the weak mixing angle $\theta_{w}$.

For $\theta_{w}=0$ we observe from (14) that the 2-dimensional energy $\epsilon(\mu, \nu)$ of the $Z$-NCS has a manifest maximum at $\mu=\nu=0$ and the minimax procedure over the NCS (91) leads precisely to the $Z$-string (18), with $f_{Z}=\bar{f}$ and $h_{Z}=\bar{h}$. The $Z$-string solution, for $\theta_{w}=0$, has thus at least two negative modes. By continuity, we expect the same conclusion to hold for small enough values of $\theta_{w}$. This can be investigated with the general ansatz (9) for the Z-NCS.

From the energy density (14) we find, using the axial functions of the $Z$-string $f=f_{Z}$ and $h=h_{Z}$, the energy profile over the $Z$-NCS for $0 \leq[\mu \nu]<\pi / 2$

$$
\begin{aligned}
\epsilon(\mu, \nu)= & \epsilon_{Z}\left[\cos ^{2} \mu \cos ^{2} \nu\left(1-\cos ^{2} \mu \cos ^{2} \nu \sin ^{2} \theta_{w}\right) \cos ^{-2} \theta_{w} a+\right. \\
& \left.\cos ^{2} \mu \cos ^{2} \nu b+c+d\right],
\end{aligned}
$$

in terms of the following integrals

$$
\begin{aligned}
a & \equiv \epsilon_{Z}^{-1} \int_{0}^{\infty} d \rho \rho^{-1}\left(\partial_{\rho} f_{Z}\right)^{2} \\
b & \equiv \epsilon_{Z}^{-1} \int_{0}^{\infty} d \rho \rho^{-1} h_{Z}^{2}\left(1-f_{Z}\right)^{2} \\
c & \equiv \epsilon_{Z}^{-1} \int_{0}^{\infty} d \rho \rho\left(\partial_{\rho} h_{Z}\right)^{2} \\
d & \equiv \epsilon_{Z}^{-1} \int_{0}^{\infty} d \rho \rho 1 / 4\left(M_{H} / M_{Z}\right)^{2}\left(h_{Z}^{2}-1\right)^{2},
\end{aligned}
$$

which add up to 1 . The energy $\epsilon(\mu, \nu)$ for $\pi / 2 \leq[\mu \nu] \leq \pi$ decreases monotonically with $[\mu \nu]$, as follows by inspection of (15). Expanding (22) around the $Z$-string at $\mu=\nu=0$ gives

$$
\epsilon=\epsilon_{Z}\left[1+\left(\mu^{2}+\nu^{2}\right)\left(\tan ^{2} \theta_{w}-1-b / a\right) a+\mathrm{O}\left(\mu^{4}, \nu^{4}, \mu^{2} \nu^{2}\right)\right] .
$$

Since $a$ and $b$ are both positive definite we have in (24) manifest instability as long as $\tan ^{2} \theta_{w} \leq 1$. For these parameter values we can also verify from (22) that the local maximum at $\mu=\nu=0$ is in fact a global maximum of the NCS. The $Z$-string, for $\theta_{w} \leq \pi / 4$ and arbitrary Higgs mass, has thus at least two negative modes. This 
applies, in particular, to the physical mixing angle $\theta_{w} \sim \pi / 6$. In short, the $Z$ string solution of the electroweak interactions is the 2-dimensional sphaleron of a non-contractible sphere. [3

Numerical results allow us to extend the range of instability beyond $\theta_{w}=\pi / 4$ $\left(\sin ^{2} \theta_{w}=1 / 2\right)$. In table 2 we present some numerical data for the integrals (23), together with the resulting maximum value $\theta_{w}^{\mathrm{NCS}}$ for instability, as follows from (24). For $\theta_{w}<\theta_{w}^{\text {NCS }}$ the $Z$-string solution has two negative modes, made explicit by the fields (9) of the $Z$-NCS. This range of instability is consistent with the results of ref. [3]. Finally, we remark that the results of table 2 may be used to map out the complete energy profile (22) of the non-contractible sphere through the $Z$-string, as a function of both $\theta_{w}$ and $M_{H} / M_{Z}$. For the case of perturbative stability [3] of the $Z$-string, we find an energy barrier for decay towards the vacuum, which is of the order of the energy $\epsilon_{W}$ of the $W$-string.

\section{Summary}

We have constructed in this article two non-contractible spheres, which are related to certain string-like classical solutions, the so-called $W$ - and $Z$-strings. It is perhaps surprising that there are two non-contractible spheres, since the mapping $\mathrm{S}^{3} \rightarrow$ $S U(2)$ with unit winding number is essentially unique. If there were only $S U(2)$ gauge fields the non-contractible sphere would indeed be unique (up to global gauge transformations), but this is not the case when the Higgs and $U(1)$ gauge fields are included. For the second non-contractible sphere, in particular, the interplay of these fields is quite subtle in keeping the 2-dimensional energy $\epsilon$ down, for small enough values of the weak mixing angle $\theta_{w}$.

We present in fig. 1 a schematic picture of configuration space. As far as the $W$-string solution is concerned, the situation is quite simple. The $W$-string is, for all values of $\theta_{w}$, the sphaleron of a non-contractible sphere (3). The solution has thus two (or more) negative modes. As far as the $Z$-string solution is concerned, the situation is more complicated. Starting from $\theta_{w}=0$, also the $Z$-string is the sphaleron of a non-contractible sphere (9). For increasing values of $\theta_{w}$ the "balloon" of the non-contractible sphere tilts out of the $S U(2)$ plane and the $Z$-string moves away in configuration space, in order to keep its energy $\epsilon$ constant, and ultimately, for $\theta_{w}=\pi / 2$, its $S U(2)$ gauge field content vanishes altogether. As $\theta_{w}$ approaches $\pi / 2$

\footnotetext{
${ }^{3}$ Note that this result is different from the heuristic connection between the $Z$-string and the 3-dimensional sphaleron $\mathrm{S}$ as discussed in ref. 6.
} 
the negative modes of the $Z$-string turn over and the solution becomes metastable. However, for the physical mixing angle $\theta_{w} \sim \pi / 6$ the $Z$-string solution is still a genuine sphaleron, with two (or more) negative modes.

One of us (F. R. K.) thanks T. Vachaspati for interesting him in electroweak strings, the Aspen Center for Physics for hospitality and the Leids KerkhovenBosscha Fonds for a travel grant. We both thank E. Faverey for drawing the figure. 


\section{References}

[1] H. Nielsen and P. Olesen, Nucl. Phys. B61 (1973), 45

[2] N. Nambu, Nucl. Phys. B130 (1977), 505

[3] M. James, L. Perivolaropoulos and T. Vachaspati, Nucl. Phys. B395 (1993), 534

[4] M. Hindmarsh, Phys. Rev. Lett. 68 (1992), 1263

[5] T. Vachaspati, Phys. Rev. Lett. 68 (1992), 1977

[6] M. Barriola, T. Vachaspati and M. Bucher, Phys. Rev. D to be publ.

[7] F. Klinkhamer and N. Manton, Phys. Rev. D30 (1984), 2212

[8] F. Klinkhamer, Nucl. Phys. B410 (1993), 343 


\begin{tabular}{lccccccc}
\hline \hline & \multicolumn{7}{c}{$M_{H} / M_{Z}$} \\
& $1 / 8$ & $1 / 4$ & $1 / 2$ & 1 & 2 & 4 & 8 \\
\hline $\sin ^{2} \theta_{w}=0$ & 0.47 & 0.59 & 0.76 & 1.00 & 1.34 & 1.79 & 2.35 \\
& 0.34 & 0.39 & 0.44 & 0.50 & 0.56 & 0.63 & 0.69 \\
& 0.66 & 0.61 & 0.56 & 0.50 & 0.44 & 0.37 & 0.31 \\
& & & & & & & \\
$\sin ^{2} \theta_{w}=1 / 4$ & 0.49 & 0.62 & 0.80 & 1.06 & 1.43 & 1.90 & 2.47 \\
& 0.35 & 0.40 & 0.46 & 0.52 & 0.58 & 0.65 & 0.71 \\
& 0.65 & 0.60 & 0.54 & 0.48 & 0.42 & 0.35 & 0.29 \\
& & & & & & & \\
$\sin ^{2} \theta_{w}=1 / 2$ & 0.52 & 0.67 & 0.87 & 1.16 & 1.55 & 2.06 & 2.65 \\
& 0.36 & 0.42 & 0.47 & 0.54 & 0.60 & 0.66 & 0.72 \\
& 0.64 & 0.58 & 0.53 & 0.46 & 0.40 & 0.34 & 0.28 \\
& & & & & & & \\
$\sin ^{2} \theta_{w}=3 / 4$ & 0.59 & 0.76 & 1.00 & 1.34 & 1.79 & 2.35 & 2.97 \\
& 0.39 & 0.44 & 0.50 & 0.57 & 0.63 & 0.70 & 0.75 \\
& 0.61 & 0.56 & 0.50 & 0.43 & 0.37 & 0.30 & 0.25 \\
\hline \hline
\end{tabular}

Table 1: Numerical results for the $W$-string solution : upper, middle and lower entries stand for, respectively, the dimensionless energy $\epsilon_{W}$, the integrals $\tilde{a}+\tilde{b}$ and the integrals $\tilde{c}+\tilde{d}$.

\begin{tabular}{lccccccc}
\hline \hline & \multicolumn{8}{c}{$M_{H} / M_{Z}$} \\
& $1 / 8$ & $1 / 4$ & $1 / 2$ & 1 & 2 & 4 & 8 \\
\hline$\epsilon_{Z}$ & 0.47 & 0.59 & 0.76 & 1.00 & 1.34 & 1.79 & 2.35 \\
$a$ & 0.16 & 0.18 & 0.20 & 0.21 & 0.21 & 0.20 & 0.18 \\
$b$ & 0.19 & 0.22 & 0.25 & 0.29 & 0.35 & 0.43 & 0.52 \\
$c$ & 0.50 & 0.43 & 0.36 & 0.29 & 0.22 & 0.16 & 0.12 \\
$d$ & 0.15 & 0.17 & 0.19 & 0.21 & 0.22 & 0.21 & 0.18 \\
$\sin ^{2} \theta_{w}^{\text {NCS }}$ & 0.69 & 0.69 & 0.69 & 0.70 & 0.73 & 0.76 & 0.79 \\
\hline \hline
\end{tabular}

Table 2: Numerical results for the $Z$-string solution : the dimensionless energy $\epsilon_{Z}$, the integrals $a-d$ and the critical mixing angle $\theta_{w}^{\mathrm{NCS}}$. 


\section{Figure caption}

Fig. 1 Sketch of configuration space, with vertically the energy $\epsilon$ of the 2dimensional configurations considered. The directions in configuration space of the $S U(2)$ and $U(1)$ gauge fields are indicated symbolically. The mass ratio $M_{H} / M_{Z}$ is considered fixed. For mixing angle $\theta_{w}=0$ the $S U(2)$ non-contractible sphere (9) is shown with one dimension suppressed : the "balloon", with parameter values $0 \leq[\mu \nu]<\pi / 2$, is attached by a "cord", with $\pi / 2 \leq[\mu \nu] \leq \pi$, to the vacuum $\mathrm{V}$. The maximum of this non-contractible sphere coincides with the $Z$-string solution. Further away in configuration space there is another solution ( $W$-string), which is degenerate in energy. For $\theta_{w}$ increasing towards its physical value $(\sim \pi / 6)$ the noncontractible sphere tilts in the $U(1)$ direction and the $Z$-string moves away, keeping its energy $\epsilon$ constant, whereas the maximum of the pure $S U(2)$ non-contractible sphere (3) gets a larger energy and corresponds to another solution ( $W$-string). The picture is schematic in that these two non-contractible spheres are not really "parallel", not even approximately. 
This figure "fig1-1.png" is available in "png" format from: http://arxiv.org/ps/hep-ph/9402207v1 